\title{
Speed++TM: A New Pencil Puzzle for Mental Arithmetic
}

\author{
*Tiong Kung Ming1, Ting Tiew Kiong2 \\ ${ }^{1}$ Nottingham University Business School, The University of Nottingham Malaysia Campus Selangor \\ Malaysia \\ ${ }^{2}$ School of Science and Technology Universiti Malaysia Sabah, Sabah, Malaysia \\ *KungMing.Tiong@nottingham.edu.my
}

\begin{abstract}
A new pencil puzzle, Speed $++^{\mathrm{TM}}$, was developed by combining and adapting the ideas of two popular Japanese pencil puzzles i.e. Kakuro and Numberlink. The novel characteristics of Speed++TM and its advantages are then compared to traditionally composed mental arithmetic questions used in practice and competitions by mental arithmetic associations. In particular, the new puzzles have a dynamic structure which in effect tests visual-spatial skills, memory, and concentration. The puzzles also look more appealing and are easy to produce through computer implemented algorithms. We further describe the potential of this puzzle as a more vibrant mental arithmetic challenge for mental arithmeticians and its viability for educational use in schools and brain training programs similar to Prof. Kawashima's world famous brain training program.
\end{abstract}

Keywords: Pencil puzzle, mental arithmetic, visual-spatial, memory, concentration

\section{Introduction}

Many logic pencil puzzles are invented and developed in Japan by the company Nikoli Co. Ltd. Nikoli publishes excellent puzzles such as the popular Sudoku and Kakuro. A less well know puzzle, Numberlink, is also part of the Nikoli stable. The WEB Nikoli (2012) is an excellent resource that discusses the ideas and characteristics of the puzzles in its collection as well as providing sample puzzles and tutorials to solve the puzzles. In Kakuro (Fig. 1), there are squares with a diagonal line and numerals above and/or below the diagonal line. The numeral above the diagonal line represents the sum across while the numeral below the diagonal line represents the sum downwards, both which are to be filled with numbers from 1 to 9 without repetition of numbers along the row or column.

Figure 1: An example of a Kakuro puzzle (a) with its solution (b) (Wikipedia - Kakuro, 2012)

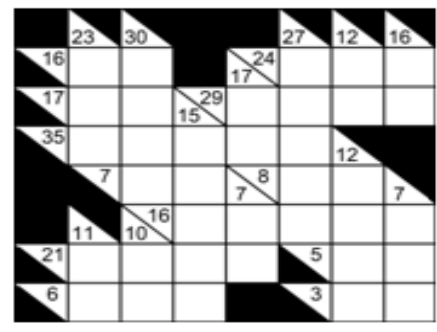

(a)

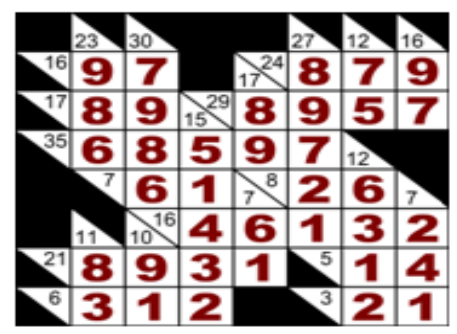

(b)

In Numberlink (Fig. 2), the objective is to link each pair of numbers with a single continuous line which passes through the squares horizontally or vertically. The lines must not cross one another and all empty squares must be used, i.e. passed by a continuous line, only once.

Figure 2: An example of a Numberlink puzzle (a) with its solution (b) (Wikipedia - Numberlink, 2012)

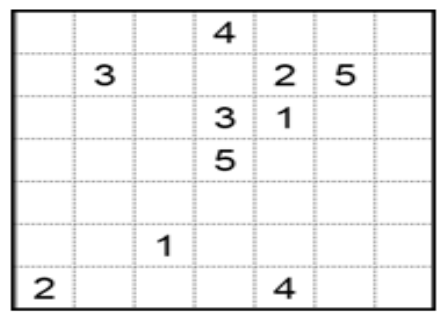

(a)

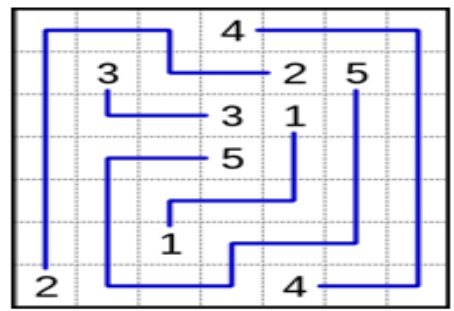

(b) 
Taking the basic ideas and concepts from Kakuro and Numberlink puzzles, we developed a new mental arithmetic based game which we named Speed++TM.

\section{The Speed++TM Puzzle}

In speed $++^{t m}$, the concepts of summation (from kakuro) and finding links (from numberlink) are purposefully combined to form a new challenging puzzle which can be applied to mental arithmeticians. In a speed $++{ }^{\mathrm{tm}}$ puzzle, the total sums are given, along with all other numbers on the grid, and the objective is to pair each of the total sums with a matching " $x$ "' with the linked paths designated by the addition of values traversed by a particular matching. No information is given regarding to which total sum (starting point) is supposed to be linked with which particular ' $x$ ' (ending point). the puzzle is considered solved when all total sums have been correctly paired with an " $\mathrm{x}$ " (fig. 3).

Figure 3: An example of a Speed $++^{\mathrm{TM}}$ puzzle (a) with its solution (b)

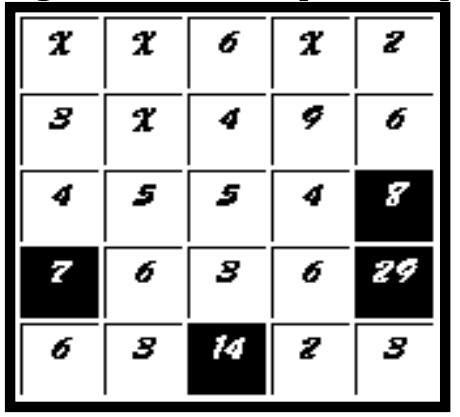

(a)

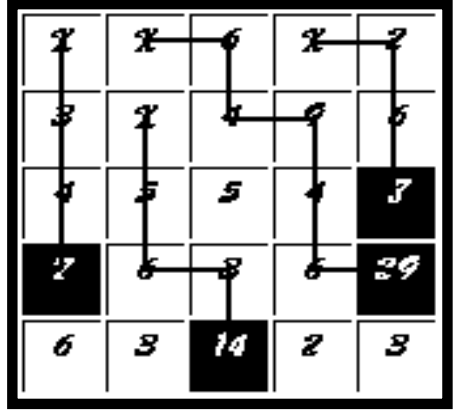

(b)

The level of difficulty of a Speed $++^{\mathrm{TM}}$ puzzle can be increased through a combination of (i) increasing the value of the total sums to be linked, (ii) increasing the size of the puzzle, and (iii) increasing the number of paired matchings (Fig. 4).

Figure 4: An example of a $11 \times 11$ Speed++ ${ }^{\mathrm{TM}}$ puzzle (a) with its solution (b)

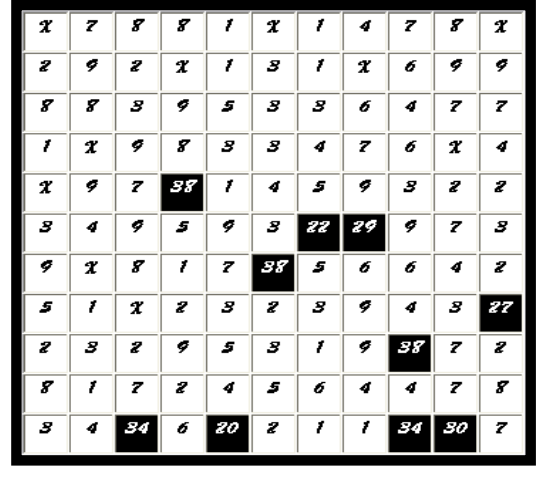

(a)

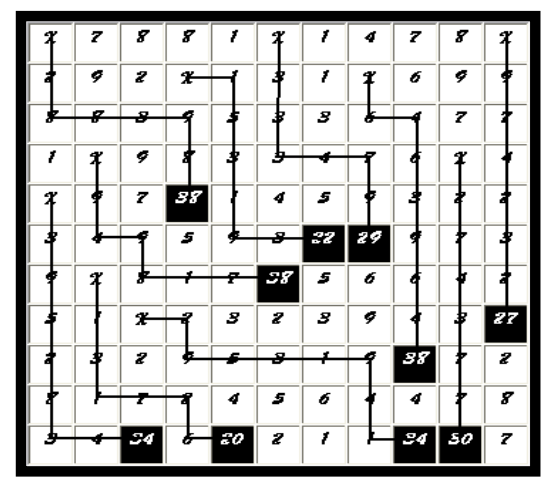

(b)

\section{Novelties and Advantages of Speed $++^{\mathrm{TM}}$}

Conceptual Innovation: In Kakuro, each sum of numbers are only found either horizontally or vertically with the length of summation known and the numbers used cannot be repeated (Fig. 1b). These characteristics render Kakuro to be a logic puzzle where it involves guessing and searching for particular combinations of numbers that fit the criteria rather than doing mental arithmetic. In Speed $++^{\mathrm{TM}}$, the summation line is a continuous line traversing both horizontally and vertically with the length of each summation not known and there can be repeating numbers in the summation. Also, there is no information on where the sums would end (i.e. which total sum is connected to which "X"). And unlike Numberlink, where only pairs of numbers are linked together, in Speed $++^{\mathrm{TM}}$ the correct summations have to be found when connecting each total sum with a particular " $X$ " each. However, unlike Numberlink, in Speed $++^{\mathrm{TM}}$ there can be leftover unused numbers in the puzzle grid (Fig. 3 and Fig. 4). For Speed++ ${ }^{\mathrm{TM}}$, a player really needs to do mental arithmetic in finding the correct pair matchings and this is where it has 
potential to be a fun and yet more challenging way to test mental arithmeticians, or rather, mental athletes.

Visual-spatial Process: Doing arithmetic is a very important basic skill and many simple math games and exercises have been devised to develop and to test arithmetic skills. However, in these games and exercises the operation is usually done the conventional way, i.e. either wholly vertically or wholly horizontally.

Figure 5: Example of vertical operation

$$
\begin{array}{r}
12 \\
3 \\
+\quad 6 \\
\hline 21
\end{array}
$$

\section{Figure 6: Example of horizontal operation}

$$
12+3+6=21
$$

In Speed++TM, the operations are still done in "one line" but the line now consists of seemingly random continuous horizontal and vertical paths. Thus, in Speed $++^{\mathrm{TM}}$, it presents a form of visual-spatial process where a player has to figure out from the available surrounding numbers what series of numbers will make the required total sums. In doing this, a player also has to avoid crossing summation lines. Additionally, in normal mental arithmetic competition questions or tests, one only requires to solve the sums in a particular section, then forget about it and move on to the next section of calculation. The length of summation is also known. Fig. 7 shows an example of mental arithmetic competition questions from Universal Concept Mental Arithmetic System, UCMAS.

Figure 7: Example of UCMAS mental arithmetic competition questions (" 7 th All India Abacus and Mental Arithmetic Competition," 2007)

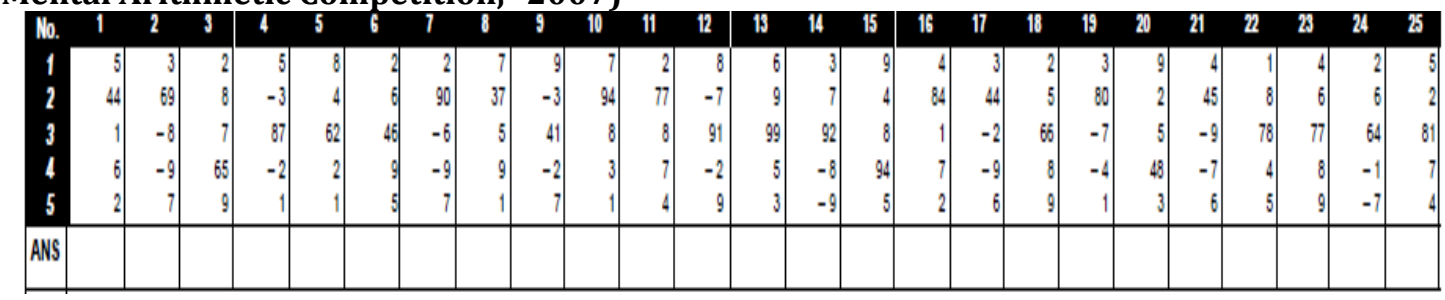

In Speed++TM however, a player would have to mentally store some of the calculation when finding the links. This requires good memory and concentration abilities. In conclusion, Speed++ ${ }^{\mathrm{TM}}$ tests a player's summation skills, visual-spatial skills, memory and concentration.

\section{Educational Use and Potential for Commercialization}

Many math learning programs claim to teach and equip learners with mental arithmetic skills or efficient arithmetic calculations through a series of techniques or continual exercises. Examples of such programs include Kumon ${ }^{\circledR}$ (http://www.kumon.com), Math Monkey ${ }^{\circledR}$ (http://www.mathmonkey.com) and UCMAS® (http://www.ucmas.com).

Ready Market: Speed++ ${ }^{\mathrm{TM}}$, we believe, is a good mental arithmetic challenge which can be used by these programs as normal classroom exercises or as test puzzles to gauge the arithmetic, visual-spatial, memory and concentration abilities of these students. Mental arithmetic competitions are actively held annually by mental arithmetic associations, e.g. UCMAS, in many nations including Malaysia and there is also an international competition (UCMAS, 2012).

New Market: Speed $++\mathrm{TM}$ is of course also suitable for any students (in fact any individual) who do not attend these math learning programs and are interested in mental arithmetic or in general, solving puzzle-type games. One important element that is being introduced in Speed $+{ }^{\mathrm{TM}}$ is the crucial element of play which is usually missing in mental arithmetic exercises or tests. This, we believe, would increase interest and participation in mental arithmetic activities. 
Evidence of Research on the Advantages of Doing Daily Simple Math Calculations: Prof. Ryuta Kawashima of Tohoku University, Japan has produced books (Kawashima, 2005, 2008) that contain many simple math calculation exercises that research has shown to improve brain function and mental age. Nintendo has even made a hugely popular game out of Kawashima's research ("Dr. Kawashima's Brain Training," 2012). We believe that Speed++TM puzzles, in particular smaller sizes like 5 X 5 to 7 X 7, are suitable for use in similar programs like Prof. Kawashima's.

Easy to Produce and Looks More Appealing: The Speed++ ${ }^{\mathrm{TM}}$ puzzles are generated by a prototype computer program (developed by the authors) in the Visual Basic language (we shall however refrain from discussing the algorithms used in generating the puzzles). The Speed $++^{\mathrm{TM}}$ puzzles can be constructed in various sizes, which present a progressive level of difficulty and challenge much like current mental arithmetic test questions. Fig. 8 shows an example of the progressive level of difficulty in conventional mental arithmetic tests questions. Contrast it with Fig. 9 which show the progressive level of difficulty in Speed $++{ }^{\mathrm{TM}}$.

Figure 8 : Example of progressive level of difficulty in conventional mental arithmetic tests (" $7^{\text {th }}$ All India Abacus and Mental Arithmetic Competition," 2007)

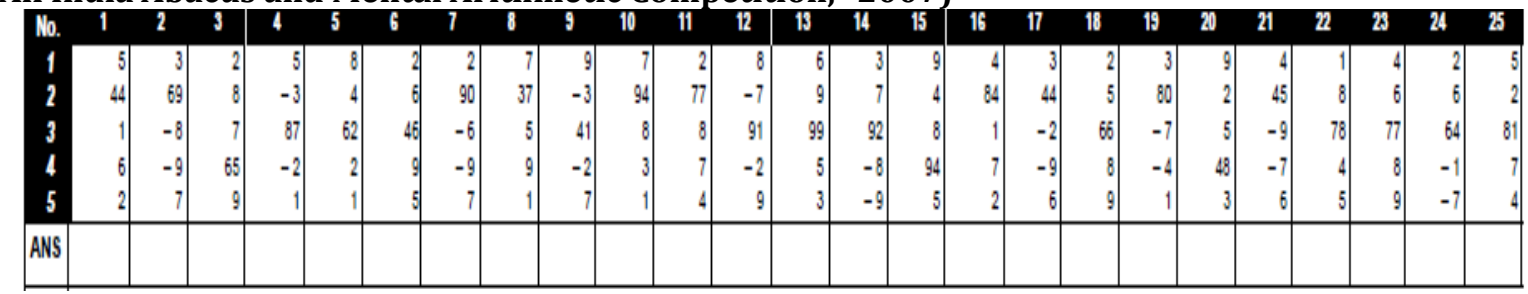

Third term, Elementary B

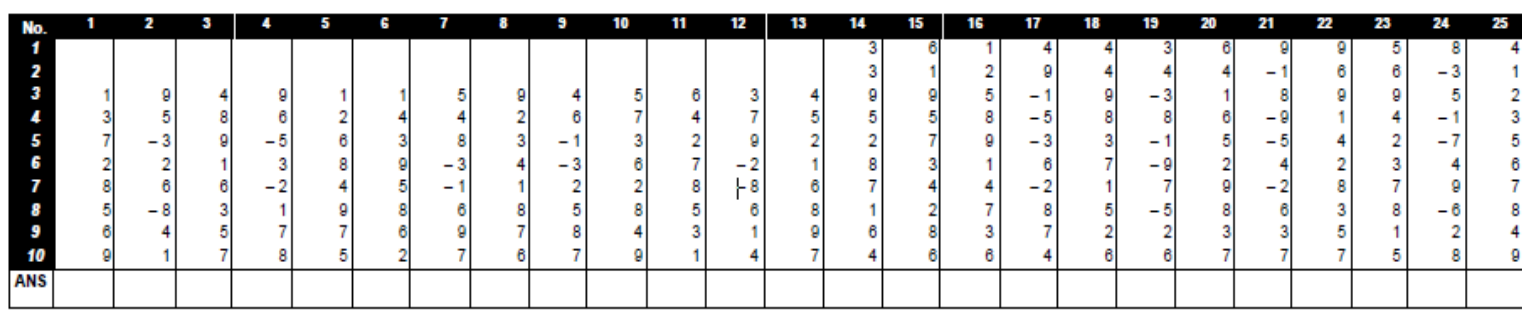

Fifth term, Intermediate B

\begin{tabular}{|c|c|c|c|c|c|c|c|c|c|c|c|c|c|c|c|c|c|c|c|c|c|c|c|c|c|}
\hline No. & 1 & 2 & 3 & 4 & 5 & 6 & 7 & 8 & 9 & 10 & 11 & 12 & 13 & & 15 & 16 & 17 & 18 & 19 & 20 & 21 & 22 & 23 & 24 & 25 \\
\hline 2 & 22 & $\begin{array}{l}80 \\
32\end{array}$ & 65 & 75 & 20 & 30 & $\begin{array}{l}85 \\
39\end{array}$ & 73 & $\begin{array}{l}12 \\
83\end{array}$ & 27 & $\begin{array}{l}27 \\
37\end{array}$ & $\begin{array}{r}6 \\
-47\end{array}$ & $\begin{array}{l}42 \\
81\end{array}$ & $\begin{array}{l}17 \\
90\end{array}$ & 35 & $\begin{array}{l}32 \\
45\end{array}$ & $\begin{array}{r}72 \\
-55\end{array}$ & $\begin{array}{l}47 \\
88\end{array}$ & $\begin{array}{l}88 \\
98\end{array}$ & $\begin{array}{r}62 \\
-43\end{array}$ & $\begin{array}{c}14 \\
52\end{array}$ & $\begin{array}{l}78 \\
62\end{array}$ & $\begin{array}{l}29 \\
96\end{array}$ & $\begin{array}{l}93 \\
17\end{array}$ & $\begin{array}{c}44 \\
50\end{array}$ \\
\hline 3 & 51 & 87 & -34 & 46 & 82 & -29 & 64 & -11 & 23 & 59 & 95 & 42 & 54 & -89 & 36 & 72 & 19 & 31 & -67 & -13 & 49 & 61 & -97 & 44 & -80 \\
\hline 4 & 52 & 34 & -39 & 44 & 26 & 31 & 37 & -19 & 24 & 29 & 11 & -1 & 22 & 92 & 98 & 14 & 85 & 90 & -72 & 77 & 82 & 64 & 70 & 75 & 57 \\
\hline 5 & 74 & 82 & 89 & 97 & 15 & -23 & 31 & -38 & 46 & 53 & 61 & 6 & 76 & -84 & 91 & 99 & -18 & 25 & -33 & -40 & 48 & 56 & -63 & 71 & -13 \\
\hline 6 & 74 & 51 & -28 & 94 & 71 & 49 & 26 & 92 & 69 & 46 & 24 & -6 & 43 & -20 & 87 & 64 & -41 & 18 & 84 & 62 & 39 & 16 & 58 & 36 & -13 \\
\hline 7 & 27 & 30 & 99 & 78 & 58 & -61 & 40 & 19 & 23 & 91 & 70 & 5 & 53 & 32 & 12 & 15 & 83 & 63 & 42 & ${ }_{45}$ & 25 & 93 & -73 & 76 & 55 \\
\hline 8 & 12 & 51 & 89 & 38 & 76 & 48 & 86 & 35 & 73 & 22 & 60 & 3 & 71 & 20 & 58 & 96 & 45 & 18 & 56 & 94 & 43 & 81 & 30 & 68 & 41 \\
\hline ANS & & & & & & & & & & & & & & & & & & & & & & & & & \\
\hline
\end{tabular}

Eighth term, Higher C

Figure 9: Example of progressive level of difficulty in Speed $++^{\mathrm{TM}}$

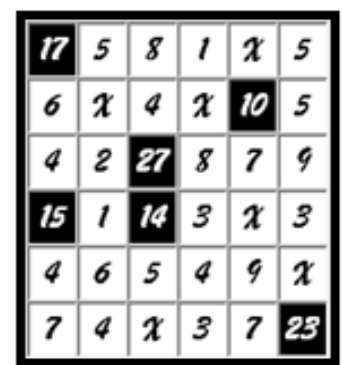

(a) $6 \times 6$

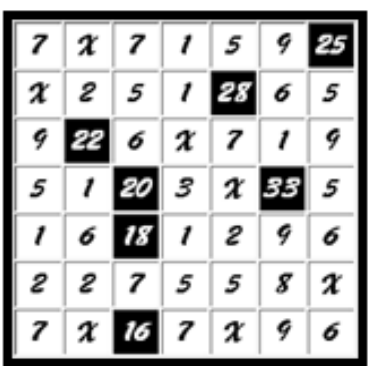

(b) $7 \times 7$

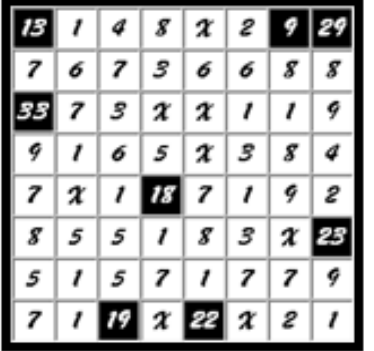

(c) $8 \times 8$ 


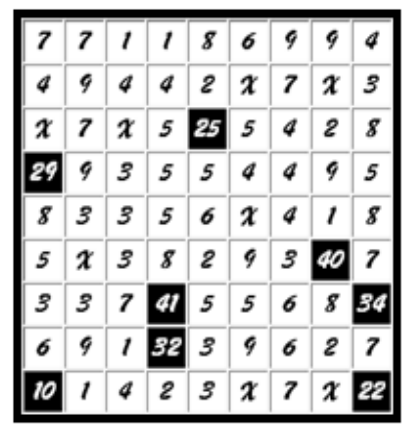

(d) $9 \times 9$

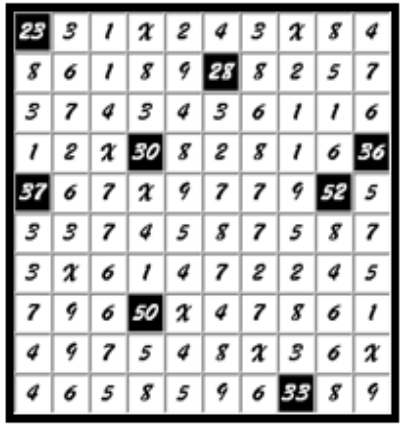

(e) $10 \times 10$

Further Work: currently, the speed++tm puzzles only involve the summation operation. it is not difficult to see that other operations (i.e. subtraction, multiplication, and division) are also implementable and the existing computer program can be tweaked to accommodate the changes. the puzzles can be designed with only one operation throughout or it could be a combination of operations in a single puzzle. the next development is to test these speed $++{ }^{t m}$ puzzles in schools and mental arithmetic programs to see the response to and the effect of these puzzles.

\section{Conclusion}

Speed $++^{\mathrm{TM}}$ is a new mental arithmetic based puzzle that introduce game play and looks more appealing to mental arithmeticians. They are relatively easy to produce and test a player's visual-spatial skills, memory, and concentration. The puzzles have potential to be used in educational settings and brain training programs.

\section{References}

$7^{\text {th }}$ All India Abacus and Mental Arithmetic National Competition (2007). Retrieved July 19, 2012, from http://www.ucmasindia.com/NationalCompetitionQuestionPaperModel.html

Dr. Kawashima's Brain Training ${ }^{\mathrm{TM}}$ : How old is your brain? (2012). Retrieved July 19, 2012, from http://www.nintendo.co.uk/NOE/en_GB/games/nds/dr_kawashimas_brain_training_how_old_is your_brain_3234.html

Kawashima, R. (2008). Train your brain more: 60 days to an even better brain, London: Penguin Books. Kawashima, R. (2005). Train your brain: 60 days to a better brain, North America: Kumon Publishing. Universal Concept Mental Arithmetic System, UCMAS (2012). Retrieved July 19, 2012, from http://www.ucmas.com/index.htm

WEB Nikoli. (2012). Nikoli puzzles. Retrieved July 19, 2012, from http://www.nikoli.co.jp/en/puzzles/ Wikipedia. (2012). Kakuro. Retrieved July 19, 2012, from http://en.wikipedia.org/wiki/Kakuro Wikipedia. (2012). Numberlink. Retrieved July 19, 2012, from http://en.wikipedia.org/wiki/Numberlink 